\title{
Low crowding agent concentration destabilizes against pressure unfolding
}

\author{
J. Somkuti ${ }^{\mathrm{a}}$, Z. Török ${ }^{\mathrm{b}}$, F. Pfalzgraf ${ }^{\mathrm{b}}$, L. Smeller ${ }^{\mathrm{b}, *}$ \\ ${ }^{a}$ Hungarian Academy of Sciences-Semmelweis University, Molecular Biophysics Research Group, Budapest, Hungary \\ ${ }^{\mathrm{b}}$ Department of Biophysics and Radiation Biology, Semmelweis University, Budapest, Hungary
}

\section{H I G H L I G H T S}

- Crowding agents stabilize against heat unfolding only above a threshold concentration.

- Crowding agents destabilize against pressure unfolding at low concentration.

- Dextran and Ficoll was used as crowding agent, BSA and lysozyme as test protein.

- Hydration volume change plays role in the pressure destabilization.

- Effect of hydration and excluded volume are discussed to explain our findings.

\section{A R T I C L E I N F O}

\section{Keywords:}

Macromolecular crowding

Ficoll

Dextran

Pressure

FTIR spectroscopy

BSA

\section{G R A P H I C A L A B S T R A C T}

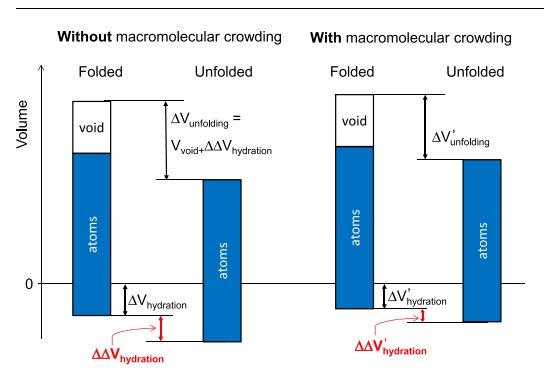

\begin{abstract}
A B S T R A C T
The concentration of macromolecules inside a cell is very high, which can affect the behavior of the enzymes, and consequently influence vital biological processes. This is called macromolecular crowding. Since the most important effect of macromolecular crowding is the excluded volume, we performed pressure experiments, where the volume (as conjugate parameter to the pressure) is the crucial factor. We measured the temperature and pressure stability of bovine serum albumin and lysozyme with various concentrations of crowding agents, dextran, Ficoll ${ }^{\mathrm{TM}}$ and lysozyme itself. Our most interesting finding is that low concentration of all the studied crowding agents decreases the pressure stability of the proteins. We explain this by the reduced hydration volume change in the crowded environment. Furthermore, we discuss the volumetric parameters and emphasize the difference between the partial volume of the protein and the volume it influences, and their relation to the excluded volume which is responsible for the macromolecular crowding.
\end{abstract}

\section{Introduction}

Although, the interior of a cell is highly crowded due to the presence of large amounts of soluble and insoluble macromolecules including proteins, nucleic acids, ribosomes, and carbohydrates; most of the in vitro protein studies have been carried out in quite dilute solutions (usually less than $1 \mathrm{mg} / \mathrm{ml}$ ). The question arises: to what extent can these properties be extrapolated to the crowded environment of the cell?
Crowding in the cell means that a significant fraction of the cell's volume is not available to other macromolecules. The concentration of macromolecules in the cytoplasm is in the range of $80-400 \mathrm{mg} / \mathrm{ml}$ $[1,2]$. In physiological fluids all macromolecules collectively occupy $10 \%$ to $40 \%$ of the total volume [3]. Although the effect of this "macromolecular crowding" is obvious, but often underappreciated. Recently, an increasing amount of evidence suggests that proteins may behave quite differently in this crowded environment. A great impact of this crowding phenomenon was demonstrated on the thermodynamics

\footnotetext{
* Corresponding author at: Department of Biophysics and Radiation Biology, Semmelweis University, Túzoltó u. 37-47, PF 263, H-1444 Budapest, Hungary.

E-mail address: somkuti.judit@med.semmelweis-univ.hu (J. Somkuti).
} 
and kinetics of many biological processes, including protein binding, folding, and aggregation.

Crowding induced stabilization of the native state of the protein was observed in several cases. This was explained by the fact that the native state is more compact. Presence of the crowding agent increased the unfolding temperature of phosphoglycerate kinase [4]. Similarly the heat denaturation temperature of hen egg white lysozyme at $\mathrm{pH} 2$ was increased by $1-3{ }^{\circ} \mathrm{C}$ depending on the crowding agent concentration [5]. The most pronounced effect was observed in case of flavodoxin, where the presence of $400 \mathrm{mg} / \mathrm{ml}$ crowding agent increased the thermal unfolding temperature by $20{ }^{\circ} \mathrm{C}$ [6].

The influence of the crowded environment on the aggregation and fiber forming of proteins depends on several factors. This can explain the variety of the experimental findings. Seelinger et al. studied the fibrillation of the type-2 diabetes mellitus related human islet amyloid polypeptide. Stabilization of the monomeric form and consequently suppression of the fibrillation was found [7]. On the contrary the addition of high concentrations of different polymers dramatically accelerated alpha-synuclein fibrillation in vitro depending on the nature, length and concentration of the polymer [8]. Crowding enhances aggregation propensity of several proteins like apoflavodoxin, creatine kinase $[9,10]$, but almost completely inhibits amyloid formation of lysozyme and stabilizes lysozyme activity $[1,11]$. The paradoxical results can be understood probably by recalling, that aggregation needs some kind of destabilization of the protein. Native proteins do not aggregate, only intermediate structures do so, which are mostly partially unfolded. Crowding seems to increase the aggregation of these destabilized structures, but their formation from the native state is presumably suppressed by crowding. This can be rationalized by the bigger volume occupied by the destabilized intermediaries.

Enzymatic activity can also be influenced by crowding. Activity of phosphoglycerate kinase was increased in presence of crowding agents. The explanation is that the active conformation is the more compact one, which was favored by the crowded environment [4].

Crowding induced stabilization can be explained by the excluded volume effect. The excluded volume is the volume, which is not available for the central point of the test molecule, because other molecules exclude it from this area [12] (Fig. 1).

According to the excluded volume effect theory, any reaction that amplifies the available volume will be stimulated by macromolecular crowding [13]. It was suggested that crowding may have a stabilizing effect on the folded protein indirectly, because the native state is more compact. Therefore, the general belief was that crowding enhances protein stabilization against denaturation by heat, cold or chemical denaturants. However, as it was pointed out recently, macromolecular crowding has both positive and negative effects on protein folding, structure and stability. The negative effect is represented by promotion of aggregation and amyloid formation [2]. These negative effects of the macromolecular crowding can be understood by considering that a fibrous state may also be more compact than the native one, which leads to favoring aggregation over correct folding [14].

Experimentally crowding is studied in vitro mostly by adding high concentration of crowding agents to the dilute solution of the target protein. These crowding agents can be hard particles like dextran or relatively open structures like Ficoll [13]. Another possibility is to use proteins (other than the test protein) [7]. The aim is to select a crowding agent, which does not have any specific interaction with the test protein, and allows measuring purely the excluded volume effect.

One of the most widely used crowding agents is Ficoll ${ }^{\mathrm{TM}}$, which is a copolymer of sucrose and epichlorohydrin. It is uncharged, highly soluble in water and commonly used to adjust density and viscosity of solutions [15]. Dextran is a polymer of D-glucopyranose. It is quite flexible and linear with less than $5 \%$ branching.

Despite of the large number of chemical, biochemical, and biophysical investigations performed as a function of temperature, the pressure is still a less known and rarely used thermodynamic parameter. Pressure as a thermodynamic parameter is conjugated to volume. This means, that pressure experiments provide more insightful information compared to temperature or chemically-induced conformational changes, since pressure induced effects can be related to volume changes [16-20].

Pressure favors the conformation with smaller volume [17,21]. Therefore, it is important to understand how the volume of a protein is composed. In case of proteins in a solution, the partial volume of the protein is defined as the volume increase of the system due to insertion of a small amount of a protein over the number of moles of the added protein [16]:

$v_{\text {protein }}=\left.\left(\frac{\partial V}{\partial n}\right)\right|_{n j, p, T}$

here $V$ is the volume of the solution and $n$ is the number of moles of the solute added [16].

This implies that the volume of the protein molecule cannot be obtained simply as the interior of a certain compartment, but it is composed of three factors:

$V_{\text {protein }}=V_{\text {atom }}+V_{\text {void }}+\Delta V_{\text {hydration }}$,

where the two first terms are volumes of the atoms and of the voids in the interior of the folded protein $[16,22]$. The latter is due to incomplete packing of the side chains. The third term is associated with the interaction of the protein with the solvent. It is known that the solvent has altered density in the surrounding of the protein. This tightly packed layer is only a few angstroms in size [23]. The volume of water in this shell is smaller than the volume of the same amount of
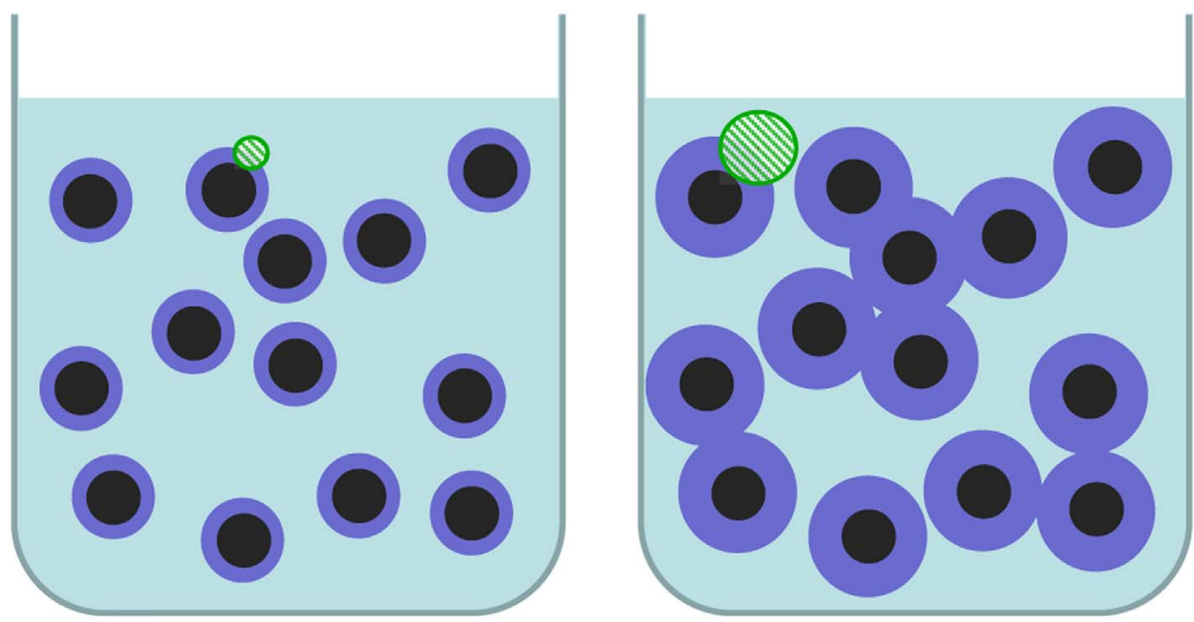

Fig. 1. The excluded volume effect. The crowding agent molecules are indicated by black circles. The black circles and the gray (pink) rings around them together represent the excluded volume. The test protein is shown by a striped circle. As the size of the test protein increases the excluded volume increases also considerably. (For interpretation of the references to colour in this figure legend, the reader is referred to the web version of this article.) 
water molecules in the bulk phase. This leads to the remarkable feature of $\Delta V_{\text {hydration }}$ namely that it gives a negative contribution.

Considering the pressure effect on proteins, three major effects can be observed depending on the magnitude of the pressure. Elastic effects already appear at the smallest pressure values. These are reversible distortions of the primary and secondary bonds. The compression of the primary chemical bonds is very small; their contribution to the volume change of the system is negligible. Compression of the hydrogen bonds can lead to the distortion of the molecular conformation, which can reduce the size of the internal cavities in the protein.

Typically if the pressure reaches $200 \mathrm{MPa}$ the intermolecular interactions and the tertiary structure are destabilized [24-26].

Higher pressure can unfold the proteins. The typical pressure required for unfolding is around $500 \mathrm{MPa}$ but this varies from protein to protein, in the range of $100 \mathrm{MPa}$ to $1 \mathrm{GPa}$ or even higher pressures in special cases [27]. The voids disappear if the protein unfolds. Since the $\Delta V_{\text {hydration }}$ is proportional to the surface of the protein, the absolute value of this term increases too, which means this term becomes more negative. Both the void volume and the change in the hydration volume contribute to the volume decrease at the pressure unfolding. Which one of these factors is more important, was the subject of a long debate $[28,29]$. From our perspective both of them might have their distinct role, and their relative contribution can vary according to the actual situation. Although pressure is well-known to unfold proteins, to our knowledge there are only a few studies reported on the effect of crowding on pressure-induced unfolding $[30,31]$. Wang et al. found that in crowded solutions high-pressure unfolding of staphylococcal nuclease was impeded [30]. According to the results of Zai et al. increasing concentrations of crowding agent dextran simply shifted the pressure stability curves of ribonuclease A towards higher pressures [31]. However, the net stabilizing effect was found to depend on the summarized effect of more stabilizing and destabilizing factors in the case of temperature stability. A similar delicate balance could govern the pressure stability too.

Our aim was to study, how the stability is modified by presence of crowding agents. The importance of this question is underlined by the fact, that both phenomena are governed by volume effects. However, there are also differences, since the volume occupied by the protein and the excluded volume effect caused by macromolecular crowding are of different kind, therefore their effect cannot be added simply. Our question is: what is the relationship between the volume induced pressure unfolding and the excluded volume induced stabilization of the native state.

For test proteins we have chosen relatively simple ones, with different size. Bovine serum albumin (BSA) is an average size protein with a $66 \mathrm{kDa}$ molar mass, but lysozyme is considerably smaller (14 kDa). These proteins have been well characterized by several spectroscopic techniques, and the pressure behavior of these proteins in absence of crowding agents is also known [32-35].

\section{Materials and methods}

\subsection{Sample preparation}

Albumin from bovine serum (Sigma) and Lyozme VI from chicken egg white (ICN Biomedicals Inc.) were available in lyophilized forms. Proteins were dissolved at the concentration of $75 \mathrm{mg} / \mathrm{ml}$ in $1 \mathrm{M}$ BisTris (Sigma) $\mathrm{D}_{2} \mathrm{O}$ buffer prior to the measurements. The $\mathrm{pD}$ of the buffer was adjusted to 7.0 by deuterated chemicals (DCl and NaOD) under $\mathrm{N}_{2}$ stream in order to avoid hydrogen contamination. The theoretical $\mathrm{pH}$ was measured and the $\mathrm{pD}$ was calculated from the following equation: $\mathrm{pD}=\mathrm{pH}$ meter reading $+0.4[36]$.

Dextran Standard 25,000 (Sigma) and Ficoll PM 70 (GE Healthcare) were applied as crowding agents in $10 \%, 20 \%$ and $30 \%$ concentrations.

A diamond anvil cell (Diacell, Leichester, UK) suitable for high pressure infrared studies was filled with the above solutions.

\subsection{Infrared spectroscopy}

The infrared spectra were recorded with a Bruker Vertex80v (Bruker Optics, Billerica, MA) FTIR spectrometer equipped with a high sensitivity MCT (mercury-cadmium-telluride) detector. Each spectrum was collected averaging 256 scans at $2 \mathrm{~cm}^{-1}$ resolution. The infrared beam was focused on a high pressure diamond anvil cell (Diacell, Leichester, UK) using a Bruker A525 type beam condenser. The diamond cell is suitable to reach more than $1 \mathrm{GPa}$ pressure under controlled temperature conditions. The thickness of the gasket was $75 \mu \mathrm{m}$. This was indented before the measurement which resulted in an optical path length of $50 \mu \mathrm{m} . \mathrm{BaSO}_{4}$ were used as internal calibrant to determine the pressure [37]. Sample temperature was controlled by a Eurotherm controller (typ 2216e, Durrington, UK) and measured by a thermocouple (OMEGA Engineering, Stamford, CT). The heating rate was $0.2^{\circ} \mathrm{C} / \mathrm{min}$.

Basically, we performed two types of experiments: pressure experiments (increasing pressure at constant temperature) and temperature experiments (increasing temperature at constant pressure). Both experiments were performed in the diamond anvil cell, because of its small sample volume. In case of temperature studies special care was taken to keep the pressure under $1 \mathrm{kbar}$.

\subsection{Data analysis}

Infrared spectra were deconvoluted with the following parameters: Lorentzian band shape, band width $=5.2 \mathrm{~cm}^{-1}$ and resolution enhancement factor $=1.84$ using the OPUS (Bruker) software.

The pressure- and temperature-induced transitions were fitted with the following formulas, respectively, either of which can be derived from the two-state transition model:

$y(p)=a+b p+\frac{\Delta a+\Delta b p}{1+\exp \left(\frac{\left(p-p_{1 / 2}\right) \Delta V}{R T}\right)}$

for pressure transitions, and

$y(T)=a+b T+\frac{\Delta a+\Delta b T}{1+\exp \left(\frac{\Delta H}{R}\left(\frac{1}{T}-\frac{1}{T_{1 / 2}}\right)\right)}$

for temperature scans. Here $y$ is the physical parameter to be fitted (e.g. position of a certain spectral line) $a$ and $b$ are the parameters describing the linear dependence of $y(p)$ or $y(T)$ below the transition, $\Delta a$ and $\Delta b$ are the changes of $a$ and $b$ during the transition, $p_{1 / 2}$ and $T_{1 / 2}$ are the transition midpoints, all the other symbols have their usual meaning.

The unfolded ratio at a given temperature or pressure was determined from the fitted curves with the following formula:

$\boldsymbol{R}_{\mathbf{u}}(\boldsymbol{p})=(\boldsymbol{y}(\boldsymbol{p})-(\boldsymbol{a}+\boldsymbol{b p})) /(\Delta a+\Delta b p)$
$\boldsymbol{R}_{\mathbf{u}}(\boldsymbol{T})=(\boldsymbol{y}(\boldsymbol{T})-(\boldsymbol{a}+\boldsymbol{b} \boldsymbol{T})) /(\Delta \boldsymbol{a}+\Delta \boldsymbol{b} \boldsymbol{T})$

These values vary between 0 and 1 , giving 0 for a completely folded case and 1 for the fully unfolded one. At the midpoints of the transitions $\left(p_{1 / 2}\right.$ and $\left.T_{1 / 2}\right)$ they are equal to $1 / 2$.

\section{Results and discussion}

\subsection{BSA in crowded environment}

First we used BSA as a test protein to investigate the effect of the increasing concentration of different crowding agents. Fig. 2 shows the specific range of the infrared spectrum, which contains relevant information about the protein. The amide I band at $1653 \mathrm{~cm}^{-1}$ (at ambient conditions) reflects the helical structure of the protein $[38,39]$. The amide I vibration is concentrated at the backbone of the protein. The carbonyl stretching gives $85 \%$ of the energy of the amide I 


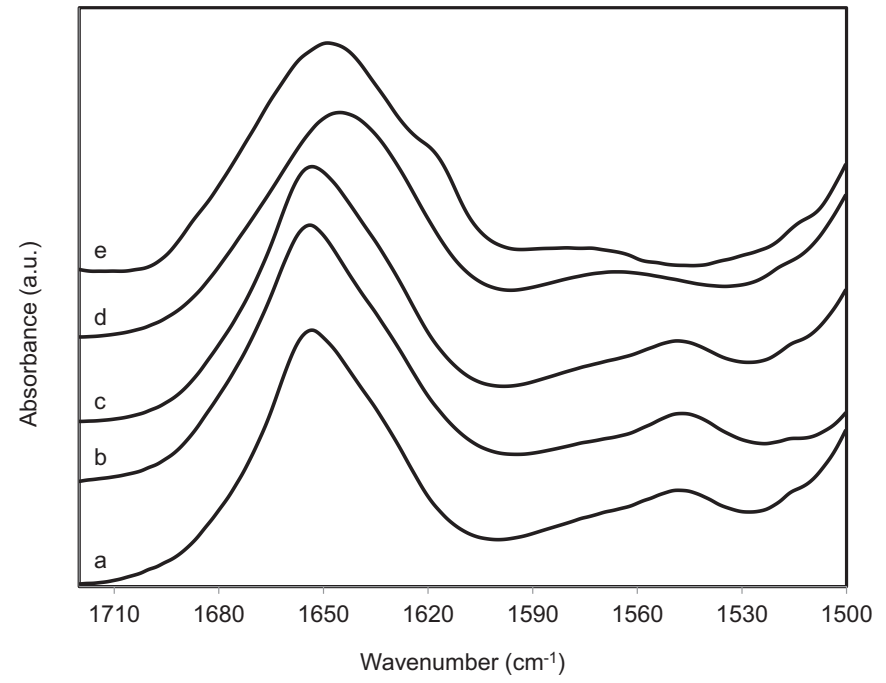

Fig. 2. Conformational sensitive range of the infrared spectrum of BSA. a) BSA without crowding agent, at $30{ }^{\circ} \mathrm{C}$, ambient pressure; b) BSA with $30 \%$ Ficoll, at $30{ }^{\circ} \mathrm{C}$, ambient pressure; c) BSA with $30 \%$ dextran, at $30{ }^{\circ} \mathrm{C}$, ambient pressure; d) BSA with $30 \%$ dextran at $30{ }^{\circ} \mathrm{C}, 10 \mathrm{kbar}(1 \mathrm{GPa})$; e) BSA with $30 \%$ dextran at $95{ }^{\circ} \mathrm{C}$, ambient pressure. The spectra are vertically shifted for the better visibility.

vibration. In a folded secondary structure, the oxygen atom of the carbonyl group takes part in a hydrogen bond. This bond perturbs the $\mathrm{C}=\mathrm{O}$ bond and consequently the amide I vibration too, which makes the amide I band very sensitive to the secondary structure. In a folded protein the amide I band is composed of several sub-bands according to the conformation of the protein. It widens and shifts to $1645 \mathrm{~cm}^{-1}$ if the protein unfolds as it can be seen in d and e curves of Fig. 2 [40,41]. Further information can be obtained from the amide II band and from the infrared absorption line of the tyrosine vibration. The amide II vibration is the bending vibration of the hydrogen atom connected to the backbone nitrogen. This can report the tertiary structure of the protein. In heavy water solutions the amide II band shifts tremendously due to $\mathrm{H} / \mathrm{D}$ exchange, when the tertiary structure loosens. If the nitrogen atom of the backbone is protonated, the amide II band appears in the range of $1545-1550 \mathrm{~cm}^{-1}$. This position is hardly dependent on the secondary structure, but it shifts to roughly $1450 \mathrm{~cm}^{-1}$ if the hydrogen is exchanged by a deuterium atom. Here it is called as amide II' band. Since our experiments have been performed in $\mathrm{D}_{2} \mathrm{O}$ buffer solutions, the amide II band is considerably reduced compared to aqueous solutions, where it is almost as intensive as the amide I band. This means that the majority of the hydrogen atoms are accessible for the solvent, and they have been exchanged already in the native state. We observed later that the remaining amide II band at $1547 \mathrm{~cm}^{-1}$ will disappear parallel with the unfolding of the protein. The small absorption band at $1515 \mathrm{~cm}^{-1}$ originates from the tyrosine ring vibrations [42]. BSA contains 20 tyrosine residues (3.4\%); most of them are hidden in the interior of the protein.

The temperature stability of BSA was investigated in the presence of two crowding agents, dextran and Ficoll. Neither of them affects the secondary structure of the protein up to a $30 \%$ concentration, as it can be seen from the spectra $b$ and $c$ of the Fig. 2 This proves the absence of any specific interactions between our test protein (BSA) and the crowding agent. Therefore, our observed effects will result from the presence of the macromolecular crowding effect and not from direct molecular contacts among the test protein and the crowding agent.

We investigated the heat unfolding of BSA systematically in the concentration range of $0-30 \%$ in the case of both Ficoll and dextran. The unfolding was followed using all the possible spectral parameters: the maximum position of the amide I band, the width of the amide I band, the amplitude of the amide II band, and in some cases also the position of the tyrosine peak. Fig. 3 shows the temperature dependence
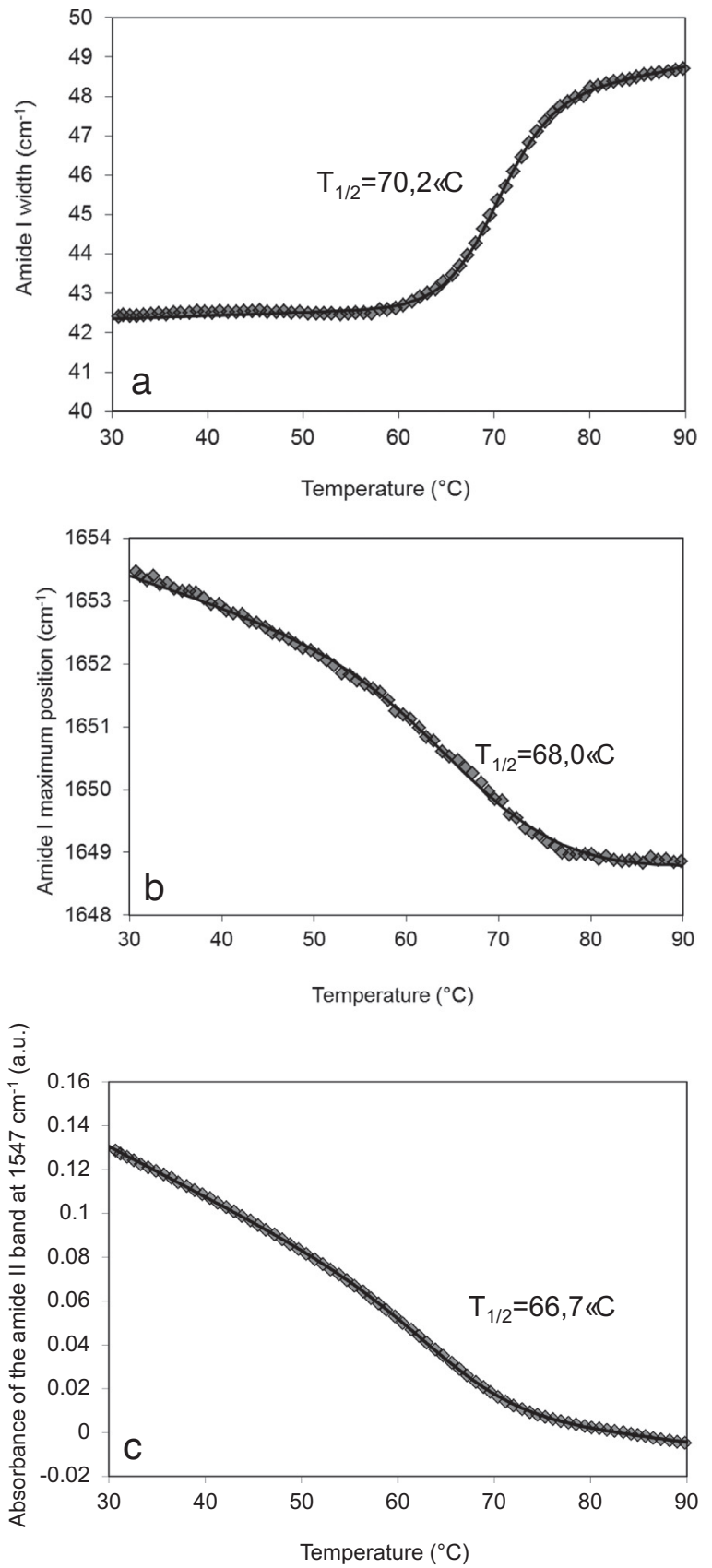

Fig. 3. Temperature dependence of the width (a) and position (b) of the amide I band and the amplitude of the amide II band (c) in a typical case (20\% Ficoll). The fitted curves (Eq. (2)) are shown as well.

of the width and position of the amide I band and the amplitude of the amide II band in a typical case (20\% Ficoll). In addition, the fitted curves (see Materials and Methods section) are plotted and the midpoint of the transition is indicated too. The fitted parameters vary slightly on the spectral parameter we fitted. This, however, is not a pitfall or an artifact; the transition temperatures show a quite logical order. The transition of the amide II is at the lowest temperature, showing that the first step in the unfolding process is the loosening of the tertiary structure. This loosening allows the hydrogen/deuterium exchange of the inner part of the protein, which was not accessible in the native conformation. The width and the position of the amide I peak are at a slightly higher temperature, but in most cases near to each other. In order to compare the transition curves obtained at different crowding agent concentrations, we normalized the spectral changes 


\section{a}

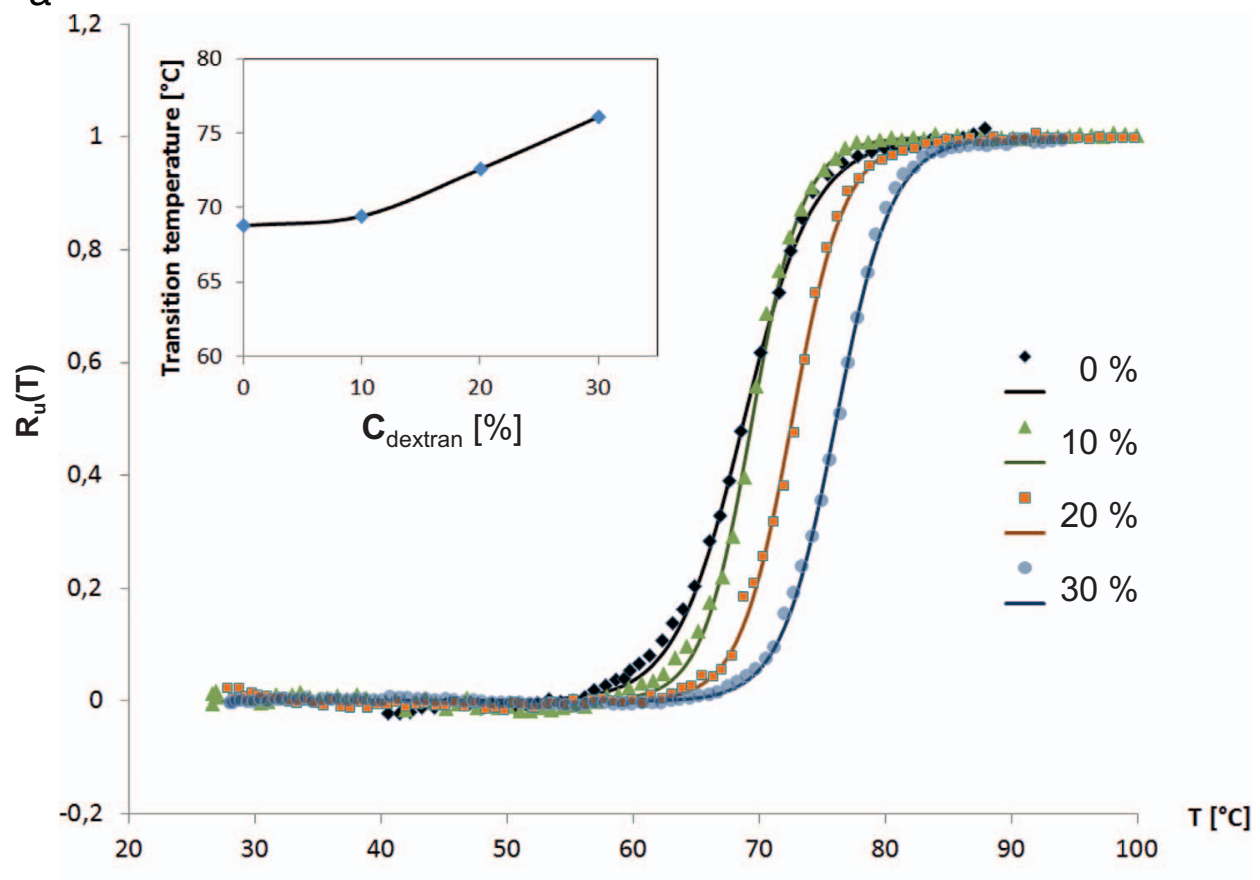

Fig. 4. Normalized transition curves of the heat-unfolding of BSA in case of increasing dextran (a) and Ficoll (b) concentrations. Inset shows the transition temperature $\left(T_{1 / 2}\right)$ vs. concentration of the crowding agent.

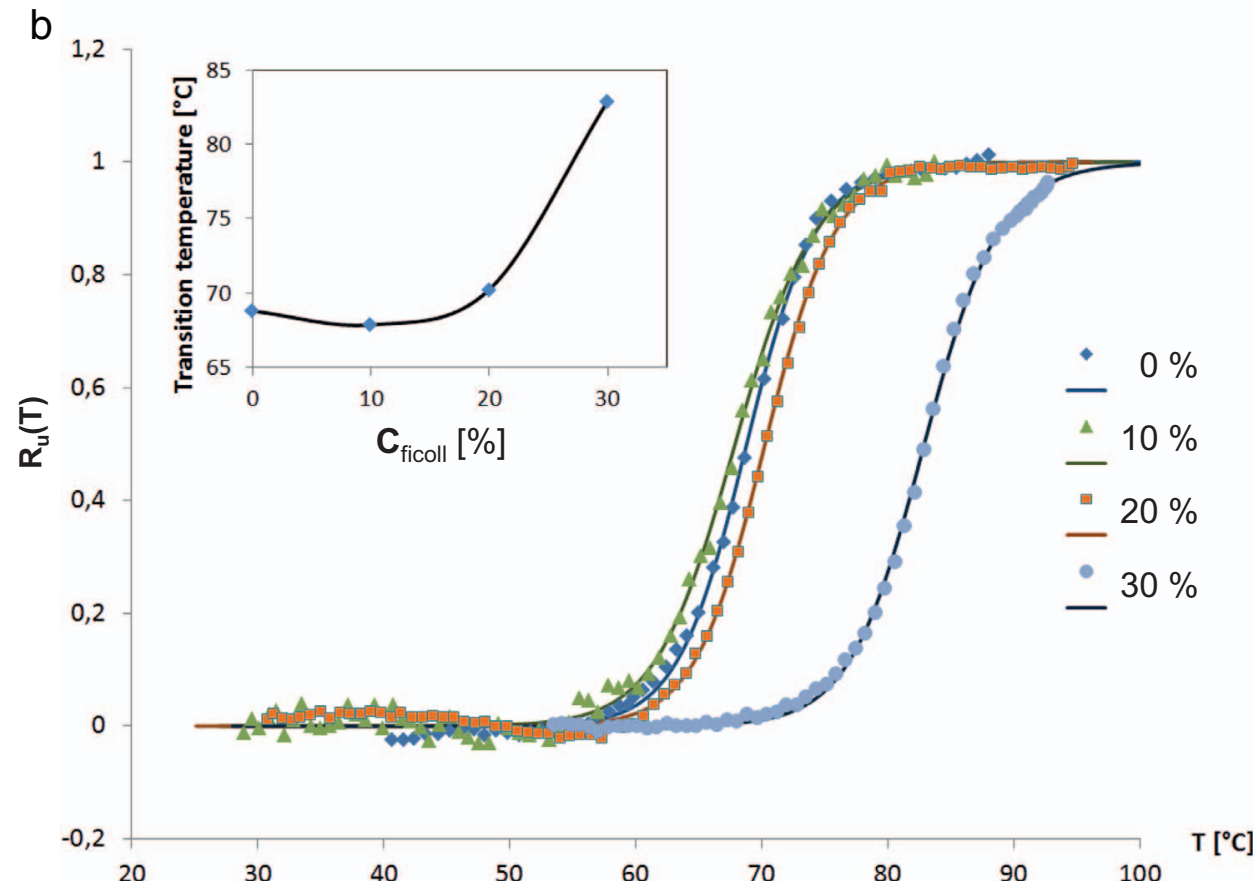

showing later only the folded ratio defined in the Materials and Methods section.

As control Ficoll and dextran solutions were measured as function of the temperature, but the infrared spectra did not show any sign for structural changes in the $20-95^{\circ} \mathrm{C}$ range (data not shown).

Fig. 4 shows the normalized transition curves of the heat-unfolding of BSA in case of increasing dextran (a) and Ficoll (b) concentrations. The high concentration of the crowding agents clearly stabilizes the protein. However, this stabilizing effect is far from being proportional to the concentration of the crowding agent. As shown on the inserts, in the case of both crowding agents a certain critical concentration is required for an active stabilization. In the case of Ficoll even a slight destabilization can be observed at low concentrations. The transition curves run almost parallel to each other; only the 0\% curve of Fig. 4a diverges from this rule, but the slopes of the curves at the transition points which can be related to the enthalpy changes $(\Delta H)$ do not differ more than $\pm 15 \%$ from each other. This means that the energetics of the transition is not significantly influenced by the presence of the crowding agent molecules. Nonlinear dependence of the stabilization on the crowding agent concentration was also observed by Sasahara et al. in case of dextran induced stabilization of lysozyme [5]. In their experiment the unfolding temperature was hardly affected by $10 \%$ dextran, but $30 \%$ crowding agent caused doubled increase of the unfolding temperature compared to $20 \%$ dextran. It is remarkable that 
a

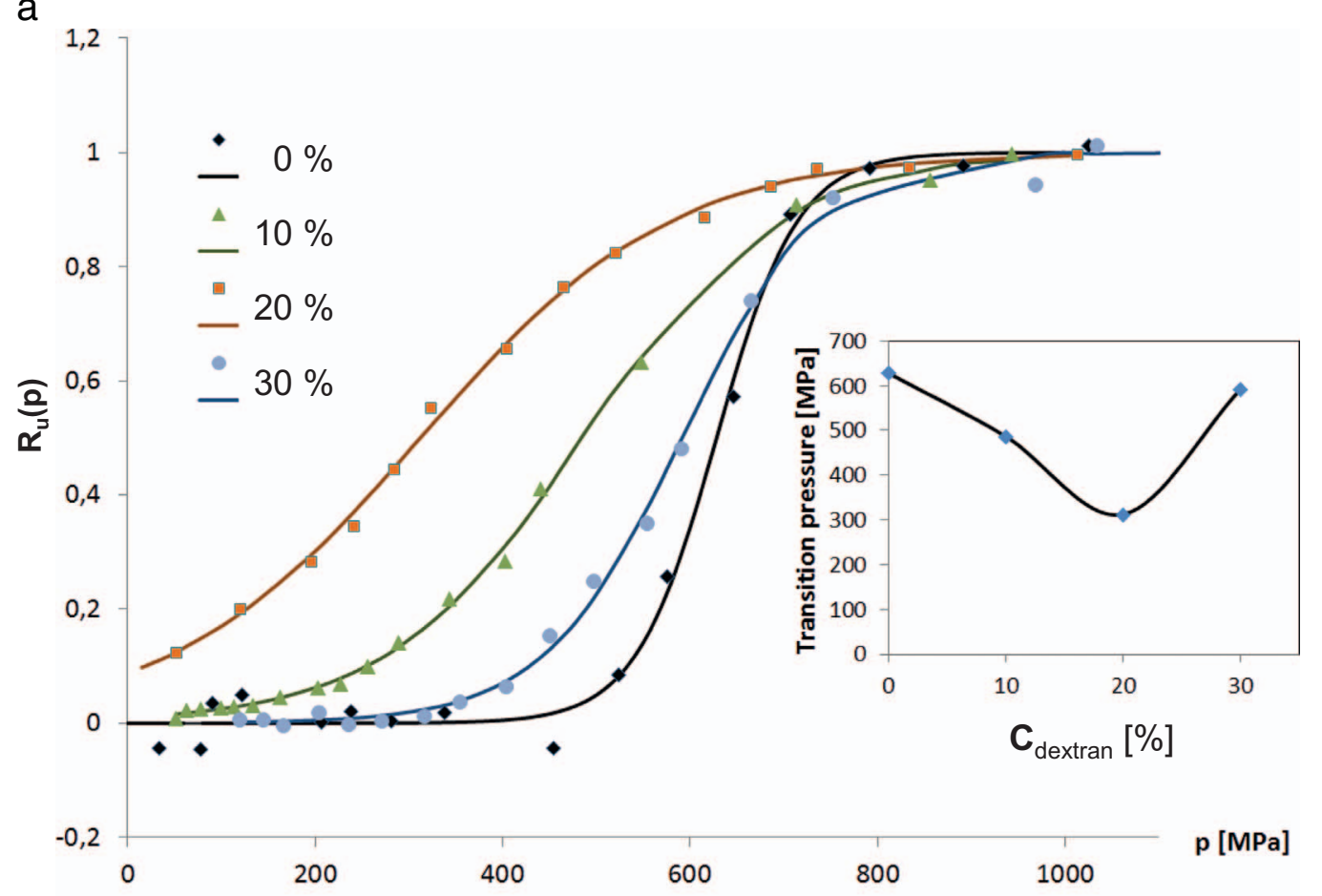

Fig. 5. Normalized transition curves of the pressure-unfolding of BSA in case of increasing dextran (a) and Ficoll (b) concentrations. Inset shows the transition pressure $\left(p_{1 / 2}\right)$ vs. concentration of the crowding agent.

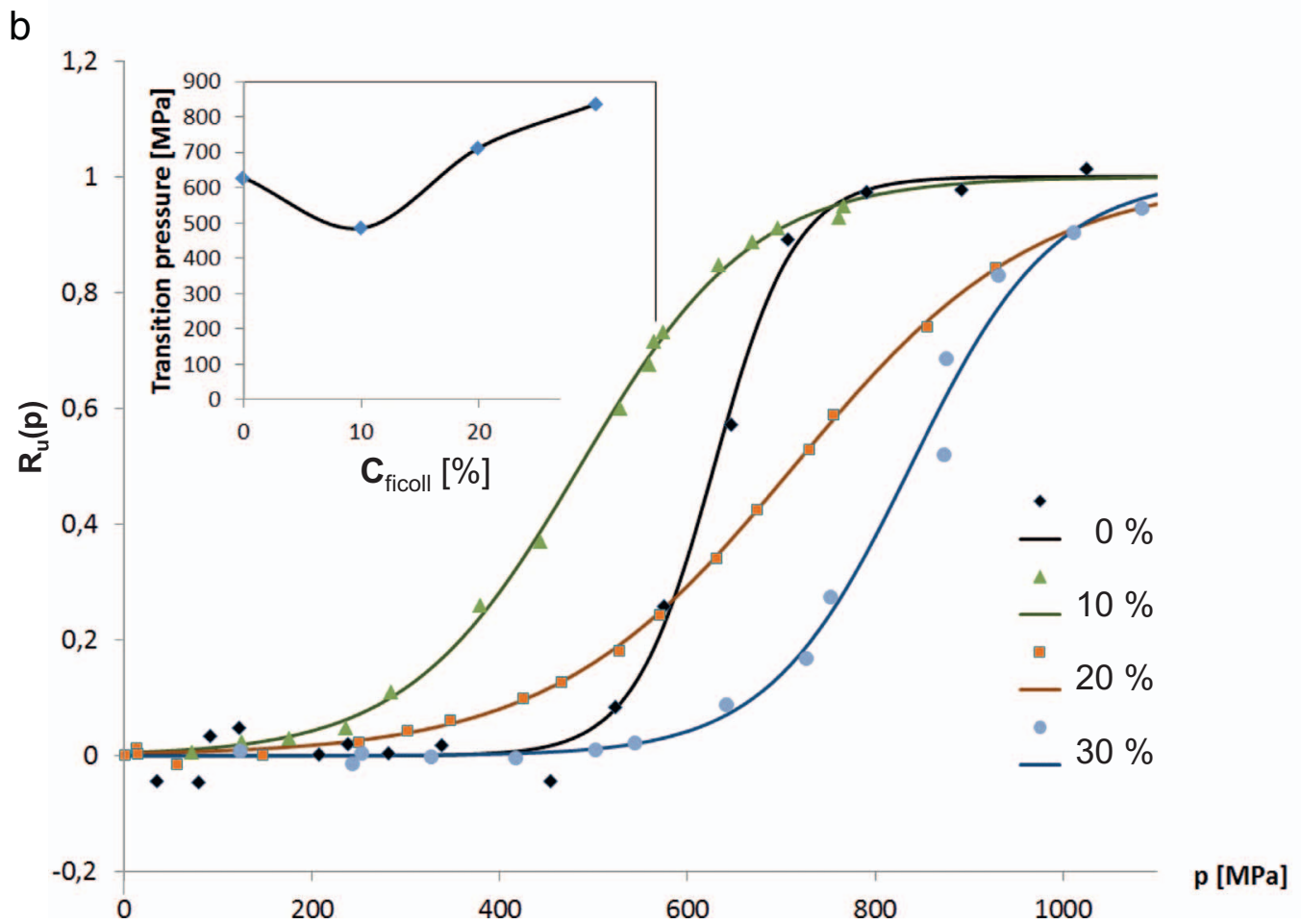

they also did not obtain significant changes in $\Delta H$ of the transition. Similar tendency was observed by Zhai and Winter: increasing the dextran concentration from $20 \%$ to $30 \%$ had almost as large increase in the temperature stability of Rnase $\mathrm{A}$ as it was caused by the concentration increase from 0 to $20 \%$.

In the case of pressure unfolding, the stability curves were even more surprising. As it is shown in the insert of Fig. 5, there is a clear concentration range, where the crowding agent causes destabilization of our target protein. Although both crowding agents managed to stabilize the protein at the highest concentration we used (30\%). The unfolding pressure was considerably lowered in the range of $10-20 \%$. This is an important finding, which to our knowledge was never observed in pressure experiments on macromolecular crowding published earlier $[30,31]$. Actually closer look to the data published by Wang et al. one can recognize, that they observed a slight destabilization (although within the experimental error level) in case of 16\% PEG. 25\% PEG caused a clear increase in the unfolding pressure in their experiments [43]. They however did not discuss this concentration effect in 


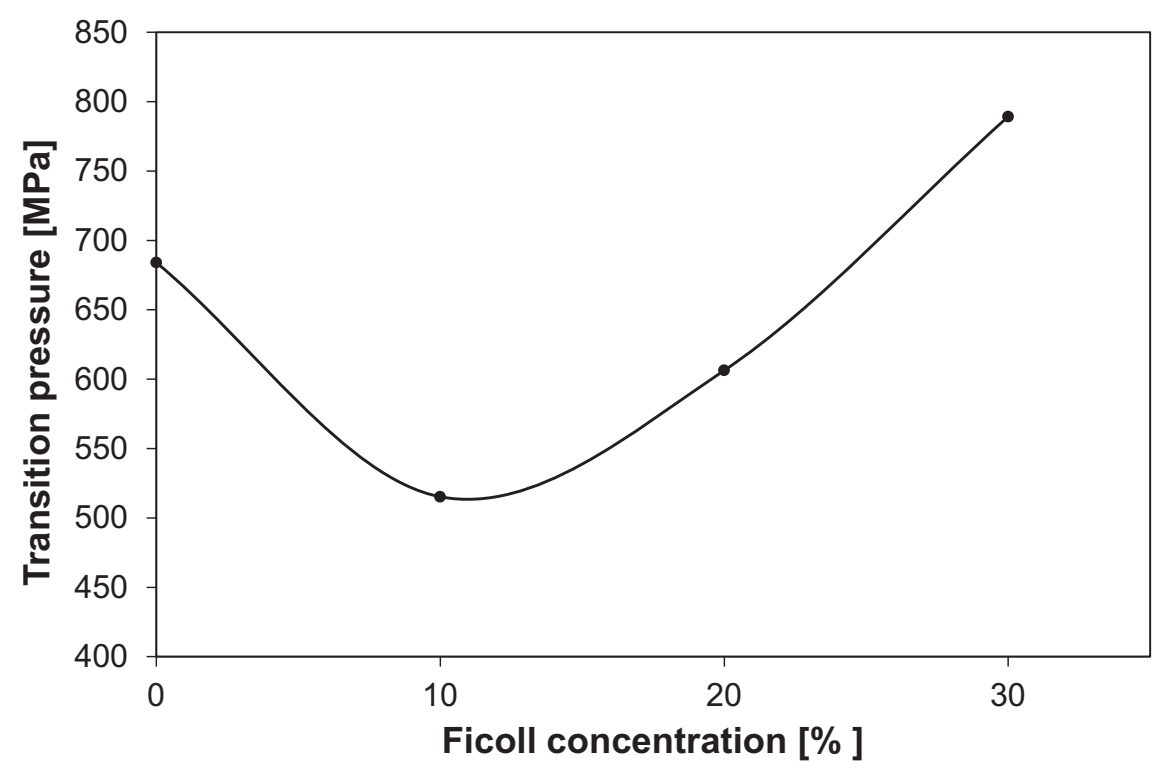

Fig. 6. The unfolding pressure $\left(p_{1 / 2}\right)$ versus Ficoll concentration in case of lysozyme. their paper.

\subsection{Crowding effect in lysozyme}

In order to see whether this u-shape destabilization curve is a rare exception or a general phenomenon, we also investigated another target protein hen egg white lysozyme. We observed a similar effect. The unfolding pressure decreases first with increasing Ficoll concentration. However, above $10 \%$ another stabilization range begins. The change of the unfolding pressure versus Ficoll concentration can be seen in Fig. 6. The similarity of this plot with the insets of Fig. 5 points towards the generality of the biphasic effect of macromolecular crowding on the pressure stability of the proteins.

The similarity between Figs. $5 \mathrm{~b}$ and 6 could raise the question whether the behavior of Ficoll is the main source of this u-shape behavior. To rule out this hypothesis, and exclude the effect of direct interaction between the crowding agent and the test molecule, we achieved crowding conditions without any foreign crowding agent molecule. This was done by using high concentrations of the test protein lysozyme. In this case both the test and the crowding agent was the same molecule. Therefore we call this situation as self-crowding. Fig. 7 shows the change in stability as function of the protein concentration. As it can be seen, the u-shape curve appears here too. This result rules out any direct interaction between the crowding agent and the test protein and shows that crowding condition itself is responsible for the u-shape curve.

\subsection{Volume and excluded volume effects in a crowded environment}

In order to explain the u-shape destabilization curve in the case of pressure unfolding, one has to consider the volume effects. As mentioned earlier, pressure acts through volume changes.

The macromolecular crowding effect is also explained frequently by the excluded volume. However, we must keep in mind the difference between excluded volume and volume. Excluded volume is the volume, which is not accessible for the (middle point of the) test molecule due to the presence of the crowding agent. It is remarkable that this volume does not equal with the volume occupied by the crowding agent. Furthermore, it also depends on the size of the test molecule, as it can be seen in Fig. 1. This is a well-defined part of the solvent. On the

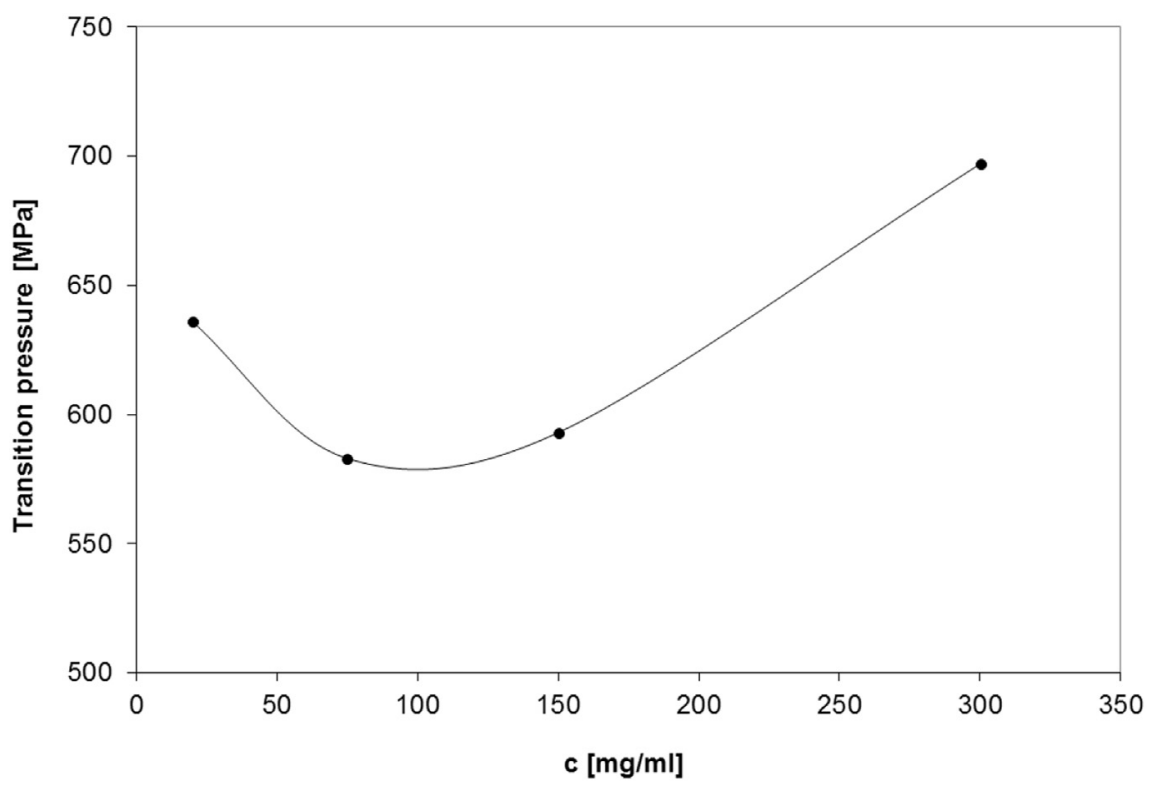

Fig. 7. Self-crowding of lysozyme. The unfolding pressure $\left(p_{1 / 2}\right)$ versus lysozyme concentration. 
contrary, the volume of the protein is not a confinable volume, but as we have seen earlier it is the volume, which is associated to the presence of the protein (see Eq. (2)).

In the case of pressure unfolding, the volume of the protein decreases, although the size of the protein might increase. This could be visually explained as follows: if we want to put the folded and the unfolded proteins both into a (microscopic, theoretical) sack, the size of the sack for the unfolded protein would be greater, because it contains a loosened protein structure and a considerable amount of water. The sack for the native protein is smaller, since the native conformation is more compact. We will call the volume of the sack as the influenced volume of the protein $\left(V_{\text {infl }}\right)$.

Assuming spherical crowding agents and test protein molecules, the excluded volume of the solution can be written on the basis of the Fig. 1 to a first approximation as:

$V_{\text {excluded }}=4 \pi\left(r_{\text {ca }}+r_{\text {inf }}\right)^{3} / 3 * N_{\text {ca }}$

where $r_{\mathrm{ca}}$ is the radius of the crowding agent, $r_{\mathrm{infl}}$ is the radius of the influenced volume of the test protein, and $N_{\text {ca }}$ is the number of the crowding agent molecules. Although, this approximation neglects the overlapping excluded volume spheres belonging to the neighboring crowding agent molecules, it is a good approximation to create a simple theory to explain the observed effects. On the other hand, the volume of the crowding agent itself is:

$V_{\mathrm{ca}}=4 \pi r_{\mathrm{ca}}{ }^{3} N_{\mathrm{ca}} / 3=V_{\text {solution }} c_{\mathrm{ca}}$

where $c_{\mathrm{ca}}$ is the concentration of the crowding agent. Combining these equations one gets the available volume:

$V_{\text {avail }}=V_{\text {solution }}-V_{\text {excl }}=V_{\text {solution }} *\left(1-c_{\mathrm{ca}} *\left(\alpha^{3}+3 \alpha^{2}+3 \alpha+1\right)\right)$

where $\alpha$ denotes the ratio of the radii of the influenced volume of the test protein and that of the crowding agent.

Zocchi's mechanical size measurements $[44,45]$ showed a $9 \mathrm{~nm}$ increase in the linear size of BSA upon chemical denaturation. This means the doubling of the original linear dimension of the protein.

Taking into account the Stokes radius of Ficoll 70, which is $5.1 \mathrm{~nm}$ and the size of BSA, which is $3.5 \mathrm{~nm}$ [46] the original $\alpha$ parameter changes from 0.7 to 1.4 . Fig. 8 shows the available volume fraction calculated above as the function of the $\alpha$ parameter at different crowding agent concentrations. As it can be seen, for $\alpha=0.7$ the available volume decreases below the partial volume of the protein (c.a.
$6 \%$ ) if the crowding agent concentration increases above $20 \%$. In the case of $\alpha=1.4$ the calculation gives a negative available volume even at $10 \%$ crowding agent concentration, which means the molecules are in contact with each other. This leads to the stabilization of the folded state of the protein at high crowding agent concentration.

On the contrary, we have a clear destabilization effect at lower crowding agent concentrations against the pressure unfolding. This will be explained by the effect of the crowding agent on the volume of the protein. As it can be observed in Fig. 9 the volume change associated to the unfolding $(\Delta \mathrm{V})$ changes also with the concentration of the crowding agent. The concentration dependence of $\Delta \mathrm{V}$ shows also a u-shape curve, similarly to the tendency obtained from the unfolding pressure values. How can the presence of a crowding agent decrease the unfolding volume? To answer this question, we have to recall the way the volume of the protein is composed of the volume of the atoms, voids and of the contraction of the surrounding water (see Eq. (2)). Since $V_{\text {atom }}$ does not change in this pressure range, only the two other terms of Eq. (2) can play a role. If the cavities were influenced by the crowding, the protein would be swollen and the loosened structure would allow the completion of the hydrogen/deuterium exchange. Since the amide II band has the same intensity in the spectra a, b and c in Fig. 2, crowding itself does not influence the folded conformation. This means that the loosening of the secondary structure and consequently any change in the void volume can be excluded. The observed volume effect can therefore occur only due to the hydration volume change. In the presence of a crowding agent the water structure can be more ordered than in the absence of a crowding agent. This phenomenon reduces the volume gain $\left(\Delta V_{\text {hydration }}\right)$ during the insertion of a protein into a solution. Similarly, the hydration volume change associated to the unfolding decreases. This is shown in Fig. 10. This phenomenon can explain the decrease in the unfolding volume change at low crowding agent concentrations. However, the increase in volume change at high crowding agent concentrations cannot be explained by this mechanism. Nevertheless, we should remember, that in this range (over 15\% crowding agent concentration) we have almost no free volume left for the test protein in the unfolded case. This implies that a direct mechanical contact with the crowding agent and a consequent mechanical deformation of the crowding agent can occur. This might lead to a slight compaction of the crowding agent structure. On the other hand, the unfolded protein is in a random flexible conformation, consequently this contact drives the protein in the direction of the more compact

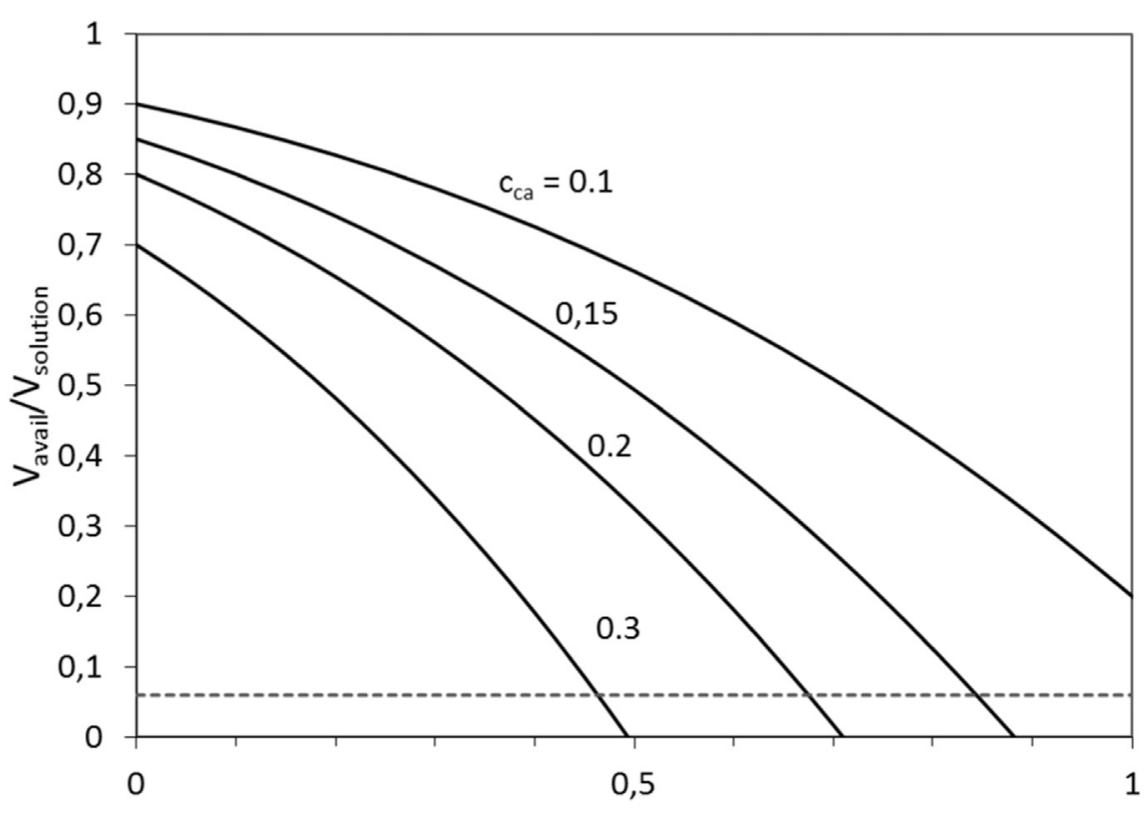

Fig. 8. Available volume fraction as function of $\alpha$ calculated from the Eq. (9). See the text for the details of the calculation. The horizontal broken line represents the volume fraction of the test protein in our experiments. 




Without macromolecular crowding

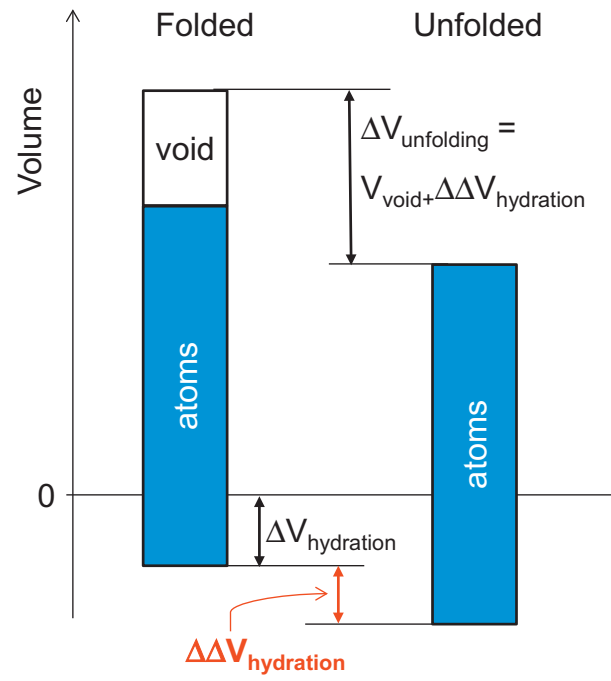

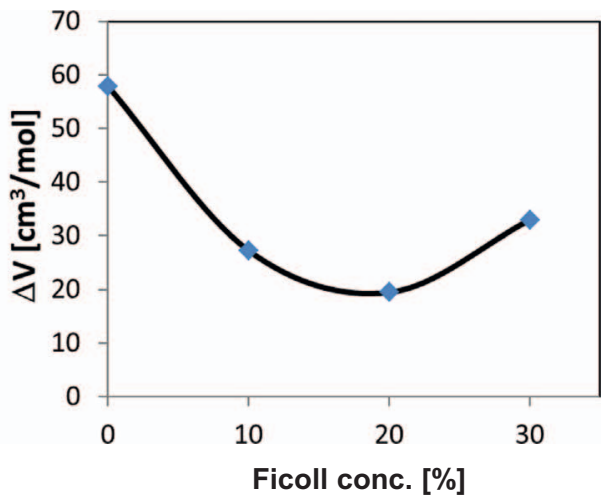

Fig. 9. Volume changes associated to the pressure unfolding of BSA vs. dextran (a) and Ficoll (b) concentration.

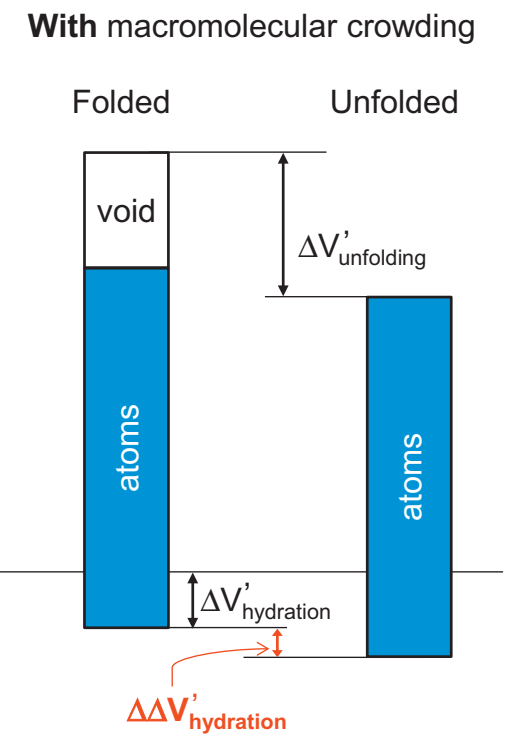

Fig. 10. Schematic explanation to the volume and volume changes of the protein without and with crowding agent. state too. This can be one of the reasons of the stabilization at high crowding agent concentrations. It is to be remembered, that the experiment measures the volume change of the total system. If the unfolding of the protein influences the crowding agent, the volume changes in the crowding molecule can be added to the volume changes of the protein. This way the unfolding volume change increases again at high crowding agent concentrations. This effect is quite similar in the case of both crowding agents.

\section{Summary}

We observed the stabilizing effect of crowding agents dextran and Ficoll on the heat unfolding, but only above certain threshold concentration. In the case of pressure unfolding, we have an initial destabilization and stabilization only at high concentrations of the crowding agents. The unfolding volume change has identical characteristics to this. This u-shape curve was explained by the counteracting effects of reduced hydration volume gain and excluded volume effect. Because the hydration state can influence the activity of proteins, the crowding conditions can influence the cellular processes via two opposite ways. Since the cell's interior is a highly crowded environment, this finding is of great relevance in understanding the essential physiological processes.

\section{Acknowledgement}

L.S. and J.S. thank to the Hungarian Research Fund OTKA 77730, L.S. Thanks to the Turchanyi Research Grant 02/16 (Hungary).

\section{References}

[1] B. van den Berg, R.J. Ellis, C.M. Dobson, Effects of macromolecular crowding on protein folding and aggregation, EMBO J. 18 (24) (1999) 6927-6933.

[2] S. Mittal, R.K. Chowhan, L.R. Singh, Macromolecular crowding: macromolecules friend or foe, Biochim. Biophys. Acta Gen. Subj. 1850 (9) (2015) 1822-1831.

[3] R.J. Ellis, A.P. Minton, Cell biology: join the crowd, Nature 425 (6953) (2003) 27-28.

[4] A. Dhar, et al., Structure, function, and folding of phosphoglycerate kinase are strongly perturbed by macromolecular crowding, Proc. Natl. Acad. Sci. U. S. A. 107 (41) (2010) 17586-17591.

[5] K. Sasahara, P. McPhie, A.P. Minton, Effect of dextran on protein stability and conformation attributed to macromolecular crowding, J. Mol. Biol. 326 (4) (2003) 1227-1237.

[6] L. Stagg, et al., Molecular crowding enhances native structure and stability of alpha/beta protein flavodoxin, Proc. Natl. Acad. Sci. U. S. A. 104 (48) (2007) 18976-18981.

[7] J. Seeliger, A. Werkmuller, R. Winter, Macromolecular crowding as a suppressor of human IAPP fibril formation and cytotoxicity, PLoS One 8 (7) (2013).

[8] V.N. Uversky, et al., Accelerated alpha-synuclein fibrillation in crowded milieu, FEBS Lett. 515 (1-3) (2002) 99-103.

[9] Y.Q. Fan, et al., Effects of macromolecular crowding on refolding of recombinant human brain-type creatine kinase, Int. J. Biol. Macromol. 51 (1-2) (2012) 113-118.

[10] R. Engel, et al., Macromolecular crowding compacts unfolded apoflavodoxin and causes severe aggregation of the off-pathway intermediate during apoflavodoxin folding, J. Biol. Chem. 283 (41) (2008) 27383-27394.

[11] B.R. Zhou, et al., Mixed macromolecular crowding inhibits amyloid formation of hen egg white lysozyme, Biochim. Biophys. Acta Gen. Subj. 1784 (3) (2008) $472-480$.

[12] A.P. Minton, Models for excluded volume interaction between an unfolded protein and rigid macromolecular cosolutes: macromolecular crowding and protein stability revisited, Biophys. J. 88 (2) (2005) 971-985.

[13] R.J. Ellis, Macromolecular crowding: obvious but underappreciated, Trends Biochem. Sci. 26 (10) (2001) 597-604.

[14] D. Homouz, et al., Crowded, cell-like environment induces shape changes in 
aspherical protein, Proc. Natl. Acad. Sci. U. S. A. 105 (33) (2008) 11754-11759.

[15] S. Mukherjee, et al., Effect of macromolecular crowding on protein folding dynamics at the secondary structure level, J. Mol. Biol. 393 (1) (2009) 227-236.

[16] K. Heremans, L. Smeller, Protein structure and dynamics at high pressure, Biochim. Biophys. Acta Protein Struct. Mol. Enzymol. 1386 (2) (1998) 353-370.

[17] J.L. Silva, D. Foguel, C.A. Royer, Pressure provides new insights into protein folding, dynamics and structure, Trends Biochem. Sci. 26 (10) (2001) 612-618.

[18] F. Meersman, et al., Structural and mechanical properties of TTR105-115 amyloid fibrils from compression experiments, Biophys. J. 100 (1) (2011) 193-197.

[19] F. Meersman, P.F. McMillan, High hydrostatic pressure: a probing tool and a necessary parameter in biophysical chemistry, Chem. Commun. 50 (7) (2014) 766-775.

[20] Y. Zhai, et al., Applications of pressure perturbation calorimetry in biophysical studies, Biophys. Chem. 156 (1) (2011) 13-23.

[21] L. Smeller, Pressure-temperature phase diagrams of biomolecules, Biochim. Biophys. Acta Protein Struct. Mol. Enzymol. 1595 (1-2) (2002) 11-29.

[22] G.A.P. de Oliveira, J.L. Silva, A hypothesis to reconcile the physical and chemical unfolding of proteins, Proc. Natl. Acad. Sci. U. S. A. 112 (21) (2015) E2775-E2784.

[23] F. Merzel, J.C. Smith, Is the first hydration shell of lysozyme of higher density than bulk water? Proc. Natl. Acad. Sci. U. S. A. 99 (8) (2002) 5378-5383.

[24] A.C. Oliveira, et al., Hydrostatic pressure as a tool to study virus assembly: pressureinactivation of viruses by formation of fusion intermediate states, High Pressure Molecular Science, vol. 358, 1999, pp. 497-513.

[25] G. Schay, et al., Allosteric effectors influence the tetramer stability of both R- and Tstates of hemoglobin A, J. Biol. Chem. 281 (36) (2006) 25972-25983.

[26] L. Smeller, P. Rubens, K. Heremans, Pressure effect on the temperature-induced unfolding and tendency to aggregate of myoglobin, Biochemistry 38 (12) (1999) 3816-3820.

[27] F. Tolgyesi, et al., Pressure activation of the chaperone function of small heat shock proteins, Cell. Mol. Biol. 50 (4) (2004) 361-369.

[28] T.V. Chalikian, R.B. Macgregor, Origins of pressure-induced protein transitions, J. Mol. Biol. 394 (5) (2009) 834-842.

[29] J. Roche, et al., Cavities determine the pressure unfolding of proteins, Proc. Natl. Acad. Sci. U. S. A. 109 (18) (2012) 6945-6950.

[30] S.T. Wang, M.W. Tate, S.M. Gruner, Protein crowding impedes pressure-induced unfolding of staphylococcal nuclease, Biochim. Biophys. Acta Gen. Subj. 1820 (7) (2012) 957-961.
[31] Y. Zhai, R. Winter, Effect of molecular crowding on the temperature-pressure stability diagram of ribonuclease A, ChemPhysChem 14 (2) (2013) 386-393.

[32] Y.O. Kamatari, et al., Conformational fluctuations of hen lysozyme investigated by high pressure NMR spectroscopy, Advances in High Pressure Bioscience and Biotechnology II, Proceedings, 2003, pp. 43-45.

[33] L. Smeller, F. Meersman, K. Heremans, Refolding studies using pressure: the folding landscape of lysozyme in the pressure-temperature plane, Biochim. Biophys. Acta Protein Proteomics 1764 (3) (2006) 497-505.

[34] K. Vogtt, R. Winter, Pressure-assisted cold denaturation of hen egg white lysozyme: the influence of co-solvents probed by hydrogen exchange nuclear magnetic resonance, Braz. J. Med. Biol. Res. 38 (8) (2005) 1185-1193.

[35] S. Savadkoohi, et al., Structural behaviour in condensed bovine serum albumin systems following application of high pressure, Food Chem. 150 (2014) 469-476.

[36] P.F. Glasoe, Citation classic - use of glass electrodes to measure acidities in deuterium-oxide, Curr. Contents/Phys. Chem. Earth Sci. 16 (1979) 12.

[37] P.T.T. Wong, D.J. Moffat, A new internal-pressure calibrant for high-pressure infrared-spectroscopy of aqueous systems, Appl. Spectrosc. 43 (7) (1989) 1279-1281.

[38] D.M. Byler, H. Susi, Examination of the secondary structure of proteins by deconvolved FTIR spectra, Biopolymers 25 (3) (1986) 469-487.

[39] L. Smeller, K. Goossens, K. Heremans, Determination of the secondary structure of proteins at high-pressure, Vib. Spectrosc. 8 (2) (1995) 199-203.

[40] J. Somkuti, et al., Different pressure-temperature behavior of the structured and unstructured regions of titin, Biochim. Biophys. Acta, Proteins Proteomics 1834 (1) (2013) 112-118.

[41] J. Somkuti, et al., Folding-unfolding transitions of Rv3221c on the pressure-tem perature plane, High Pressure Res. 33 (2) (2013) 250-257.

[42] A. Barth, Infrared spectroscopy of proteins, BBA-Bioenergetics 1767 (9) (2007) 1073-1101.

[43] S. Wang, M.W. Tate, S.M. Gruner, Protein crowding impedes pressure-induced unfolding of staphylococcal nuclease, Biochim. Biophys. Acta 1820 (7) (2012) 957-961.

[44] G. Zocchi, Mechanical measurement of the unfolding of a protein, Europhys. Lett. 35 (8) (1996) 633-638

[45] G. Zocchi, Proteins unfold in steps, Proc. Natl. Acad. Sci. U. S. A. 94 (20) (1997) 10647-10651.

[46] I. Axelsson, Characterization of proteins and other macromolecules by agarose-gel chromatography, J. Chromatogr. 152 (1) (1978) 21-32. 